\title{
La gastrulación de Epipedobates anthonyi (Anura: Dendrobatidae)
}

\author{
Paola Montenegro Larrea y Eugenia M. del Pino ${ }^{1}$ \\ Pontificia Universidad Católica del Ecuador, Escuela de Ciencias Biológicas, Laboratorio de Bio- \\ logía del Desarrollo, \\ Quito, Ecuador. 1edelpino@puce.edu.ec
}

Recibido: 04, 05, 2011; aprobado: 22, 07, 2011

RESUMEN.- Se describe la morfología externa e interna de la gástrula de la rana dendrobátida ecuatoriana, Epipedobates anthonyi. Los huevos tienen pigmento oscuro en el polo animal, su diámetro es de $2 \mathrm{~mm}$ en promedio y las posturas contienen entre 8 a 45 huevos. El tiempo requerido desde el inicio hasta culminar la gastrulación es de 1,5 días. La gástrula de $E$. anthonyi se caracterizó por la formación de un labio dorsal prominente en la región subecuatorial del embrión. El alargamiento del arquenterón estuvo retardado hasta después de terminar la gastrulación y las células que se acumularon en los labios del blastoporo formaron un prominente collar circumblastoporal. La gástrula de E. anthonyi comparte las características morfológicas observadas en otra rana dendrobátida, Colostethus (=Epipedobates) machalilla. Este estudio ha permitido una mejor comprensión de la gastrulación en ranas dendrobátidas y provee conocimientos adicionales sobre la biología de un miembro de la fauna ecuatoriana.

PALABRAS CLAVE: Colostethus (=Epipedobates) machalilla, Engystomops (=Physalaemus) randi, Epipedobates anthonyi, Gastrotheca riobambae, Xenopus laevis.

ABSTRACT.- We describe the external and internal morphology of the gástrula in the dendrobatid frog from Ecuador, Epipedobates anthonyi. Eggs have dark pigment in the animal pole, their diameter reaches $2 \mathrm{~mm}$ on average, and the egg clutch contains between 8 to 45 eggs. The time required from the beginning until the completion of gastrulation is of 1.5 days. The gastrula of $E$. anthonyi is characterized by the formation of a prominent dorsal blastopore lip in the subequatorial region of the embryo. Elongation of the archenteron is retarded until completion of gastrulation, and cells that accumulate in the blastopore lip form a conspicuous circumblastoporal collar at the end of gastrulation. The gastrula of E. anthonyi sha- 
res the morphological features observed in another dendrobatid frog, Colostethus (=Epipedobates) machalilla . This study allowed a better understanding of gastrulation in dendrobatid frogs, and provides additional knowledge about the biology of a member of the fauna of Ecuador.

KEYWORDS: Colostethus (=Epipedobates) machalilla, Engystomops (=Physalaemus) randi, Epipedobates anthonyi, Gastrotheca riobambae, Xenopus laevis.

\section{INTRODUCCIÓN}

Epipedobates anthonyi (Noble, 1921), (Dendrobatidae) (1) se distribuye en el sur-oeste de Ecuador, en las provincias de El Oro, Azuay y Loja y en el nor-oeste de Perú a altitudes entre 153-1769 m sobre el nivel del mar (2). Este estudio se inició con el objeto de complementar la información sobre la biología de esta especie debido a que $E$. anthonyi, conocida previamente como Phylobates tricolor y como Epipedobates tricolor (1) ha sido estudiada intensamente desde el punto de vista de comportamiento, mantenimiento en cautiverio y reproducción (3). El potente analgésico denominado Epibatidina fue descubierto en la piel de E. anthonyi de especímenes provenientes de dos sitios cercanos a la frontera con Perú, en el sistema fluvial del río Jubones y en los alrededores de Santa Isabel, Azuay (4). Curiosamente, se descubrió que ejemplares colectados en otras localidades carecían de este compuesto. También se encontró que la Epibatidina se perdía cuando las ranas eran mantenidas en cautiverio, lo que llevó a determinar que las toxinas de la piel de los dendrobátidos provienen de la dieta con la que estas ranas se alimentan en la naturaleza (4).
Epipedobates anthonyi deposita sus huevos sobre la tierra o en la vegetación y el macho cuida de la postura, la humedece con orina y la protege del ataque de congéneres hasta la eclosión de los renacuajos. Este período dura 14 días en promedio en esta especie y entre 19-20 días en C. machalilla $(5,6)$. Al cabo de ese tiempo, los renacuajos se adhieren al dorso del padre, y el mismo transporta los renacuajos hasta el agua en donde los renacuajos crecen y alcanzan la metamorfosis. El desarrollo acuático hasta la metamorfosis es de aproximadamente 50 a 60 días, y el estado adulto se adquiere 11 meses después (5).

Las ranas dendrobátidas tienen la ventaja de que pueden mantenerse y reproducirse en cautiverio y los métodos han sido detallados para E. anthonyi y C. machalilla $(3,6)$. Además, diferentes especies producen huevos de diversos tamaños. Así, el diámetro del huevo alcanza 1,6 mm en C. machalilla, $2 \mathrm{~mm}$ en $E$. anthonyi y $3.5 \mathrm{~mm}$ en Dendrobates auratus. En comparación el huevo de $X$. laevis tiene un diámetro de 1,2 $\mathrm{mm}$ (7). De acuerdo con estos datos, el volumen del huevo de C. machalilla es 
2,3 superior al del huevo de $X$. laevis. El huevo de $E$. anthonyi es 4,6 veces más grande y el de $D$. auratus es 25 veces superior al volumen del huevo de $X$. laevis (8). El tamaño del huevo puede relacionarse con cambios morfológicos y modificaciones de su estructura molecular. En cuanto a la morfología, se ha encontrado que la apariencia externa de la gástrula es altamente conservada en las ranas, aún cuando hay notables excepciones, como en caso de la rana Rhacophorus y en la salamandra gigante Megalobatrachus maximus en las cuales el labio ventral del blastoporo no se forma o su formación ha sido retardada (9). También la gástrula de la rana marsupial Gastrotheca riobambae ha sido modificada para desarrollar un disco embriónico. A pesar de ello, se forma un blastoporo como en otras ranas (10). Los grandes huevos, de 3,5 mm de diámetro, de la rana de desarrollo directo Eleutherodactylus coqui difieren del huevo de X. laevis, en cuanto a la estructura molecular (11). Es posible que los huevos grandes de otras ranas también difieran en su estructura molecular con $X$. laevis.

La gastrulación es un proceso de mucha importancia para el desarrollo de los vertebrados pues durante este proceso se separan las tres capas embriónicas denominadas ectodermo, mesodermo y endodermo y de esta manera se establece el plan del cuerpo. Por este motivo, la gastrulación se ha estudia- do en $X$. laevis, el pez cebra, el pollo y el ratón entre otros organismos (12). Es importante resaltar que el conocimiento de la morfología y control molecular de la gastrulación de $X$. laevis brinda una base para estudios comparativos del desarrollo embrionario temprano en ranas con adaptaciones reproductivas diversas. El análisis comparativo ha contribuido a la identificación de procesos morfogenéticos. Así por ejemplo, se encontró que la formación del arquenterón y su expansión son procesos independientes (13). Del mismo modo, se demostró que existe modularidad en la gastrulación. En particular la convergencia y extensión dorsales (CED), que transforman la forma esférica del huevo en la alargada del renacuajo, son procesos que se encuentran solapados en la gástrula de ranas de desarrollo rápido como $X$. laevis y Engystomops (=Physalaemus) randi. Por el contrario en las ranas de desarrollo lento G. riobambae y $C$. machalilla, la CED y el alargamiento del cuerpo ocurren después de la gastrulación $(7,8,14)$. El desarrollo de la placa precordal (también conocido como mesodermo de la cabeza) y del notocordio (o mesodermo del tronco) se encuentran solapados en la gástrula media de $X$. laevis. En cambio estos procesos morfogenéticos están separados en el tiempo en la rana dendrobátida $C$. machalilla $(8,15)$. Hemos iniciado el estudio de la gastrulación de ranas dendrobátidas, y en particular en 
E. anthonyi, para ampliar la base de conocimientos que permita la mejor comprensión de la gastrulación en anuros, a más de contribuir al mejor conocimiento de la fauna ecuatoriana.

\section{MATERIALES Y MÉTODOS}

Los embriones utilizados para este estudio fueron donados por el laboratorio de Herpetología de la Pontificia Universidad Católica del Ecuador y por la Mtr. M. Rivera. Las ranas adultas provinieron de Ecuador: Provincia de Azuay, localidad: Sarayunga, a $41 \mathrm{Km}$ de la población de Santa Isabel por la vía a Pasaje. Dos posturas provinieron de ranas colectadas en Ecuador: Provincia de Azuay, localidad: Santa Isabel, y Ecuador: Provincia de El Oro, localidad: El Progreso, respectivamente.

Se cronometró el tiempo requerido para el proceso de gastrulación desde la formación del labio dorsal del blastoporo hasta su cerramiento. Los embriones fueron mantenidos a temperatura ambiente del laboratorio que fluctuó entre 18 a $21^{\circ} \mathrm{C}$. La metodología para el cultivo, fijación y procesamiento de los embriones fue de acuerdo a (6). Se utilizó la tabla de estadios del desarrollo de $C$. machalilla (6) para determinar los estadios del desarrollo.

El análisis de los embriones consistió en la determinación de la morfología externa mediante microfotografía. Se realizaron bisecciones sagitales de los embriones mediante el uso de una micro-cuchilla y se procedió a analizar y fotografiar la morfología interna de la gástrula. Otros embriones fueron embebidos en agarosa al $6 \%$ y fueron seccionados a un espesor de 50-100 $\mu \mathrm{m}$ de espesor. Las secciones fueron teñidas con el tinte fluorescente Hoechst 33258 que se une al ADN y por tanto sirve para teñir los núcleos celulares. Se montó las secciones en placas portaobjeto con glicerina y fueron cubiertas con laminillas cubreobjeto. Las secciones fueron fotografiadas utilizando luz transmitida, contraste diferencial de interferencia y fluorescencia. Para la microscopía se utilizaron un microscopio estéreo Zeiss Stemi SV6 y un microscopio Zeiss Axiophot.

\section{RESULTADOS}

Número de huevos por postura y tiempo de la gastrulación.- Este estudio se basó en el análisis de 31 posturas de $E$. anthonyi obtenidas en cautiverio entre 2004 a 2011. El número promedio de huevos por postura fue de $20 \pm 9$ (se da la desviación estándar), con un rango de 8 a 45 huevos por postura. El tiempo requerido desde el inicio hasta culminar la gastrulación fue de 1,5 días.

Características externas de la gástrula.- El huevo de E. anthonyi alcanzó $2 \mathrm{~mm}$ de diámetro en promedio y se observó que tiene pigmento oscuro en el polo animal. El inicio de la gastrulación se determinó por la presencia del labio dorsal del blastoporo en la región 
subecuatorial del huevo, en el límite entre el hemisferio animal pigmentado y la región vegetal de color amarillento. A continuación se formaron los labios laterales y ventrales del blastoporo alrededor de un gran tapón de yema (Figura 1A). Conforme avanzó la gastrulación, el diámetro del tapón de yema se redujo, hasta que el blastoporo se cerró totalmente y el tapón de yema se retrajo totalmente hacia el interior. La caracterización de la morfología externa de la gástrula se facilitó por el pigmento oscuro de los labios del blastoporo y el color amarillento claro del tapón de yema.

Morfología interna de la gástrula.La cavidad del blastocele es amplia y ocupó aproximadamente el tercio superior del embrión en la gástrula temprana y media (Figura 1B). Esta cavidad se redujo en tamaño en la gástrula avanzada (Figura 1C, D). El techo del blastocele, que consistió originalmente de dos capas de células, se redujo a una monocapa de células en la gástrula media a avanzada.

Durante la gastrulación se formó una nueva cavidad, el arquenterón, o intestino primitivo. El tamaño del mismo fue pequeño durante la gastrulación (Figura $1 \mathrm{~B}, \mathrm{C})$ y se expandió notablemente en la gástrula avanzada (Figura 1D). Conforme avanzó la extensión del arquenterón, el blastocele se redujo (Figura 1B, C). Los labios del blastoporo concentraron a las pequeñas células que involucionaron durante la gastrulación y cuando el blastoporo estuvo completamente cerrado se pudo observar un collar circumblastoporal grande (Figura 1D) debido a la acumulación celular en los labios del blastoporo.

\section{DISCUSIÓN}

Comparación de la gástrula de $E$. anthonyi con otras especies.- El número de huevos de E. anthonyi de hasta 45 embriones es superior al tamaño de las posturas de la rana dendrobátida C. $m a-$ chalilla (6). El mayor número de huevos representa una característica óptima para los estudios de su desarrollo embrionario. El tiempo de 1,5 días requerido para la gastrulación en E. anthonyi es inferior al observado en $C$. machalilla, que corresponde a 2,7 días (6). Sin embargo, el tiempo de desarrollo de estos dendrobátidos es mayor que el rápido desarrollo de $X$. laevis y de la rana túngara, $E$. randi que solamente requieren 5 y 12 horas, respectivamente desde el inicio hasta completar la gastrulación. En cambio la rana marsupial Gastrotheca riobambae tiene un desarrollo mucho más lento, y la gastrulación requiere de 7 días (16). La morfología externa de la gástrula de $E$. anthonyi es similar a la gástrula de otras especies con huevos que tienen pigmento oscuro en el polo animal, como $X$. laevis, Rana pipiens y C. machalilla. Sin embargo, las características internas de la gástrula difiere grandemente de $X$. laevis como se explica a continuación. 
EI techo del blastocele.- La gástrula de E. anthonyi comparte con C. machalilla (6) el adelgazamiento del techo del blastocele hasta un grosor de una única capa de células. Este adelgazamiento va asociado con los movimientos de epíbole, que agrandan el techo del blastocele hasta cubrir totalmente al embrión, como en el caso de la rana marsupial G. riobambae (17). En contraste, el techo del blastocele consiste de dos capas celulares tanto en $X$. laevis como en los embriones de las rana túngara $E$. randi (18).

Expansión del arquenterón y alargamiento del notocordio.- La expansión del arquenterón depende de varias fuerzas morfogenéticas, entre ellas juega un papel importante la convergencia y extensión dorsales (CED), que contribuyen a cambiar la forma del cuerpo, de la esférica del huevo a la alargada del renacuajo. El arquenterón se alarga grandemente durante la gastrulación de $X$. laevis y $E$. randi $(7,18)$, en tanto que su alargamiento está retardado hasta los estadios finales de la gastrulación en $E$. anthonyi. Retardo en el alargamiento del arquenterón también ocurre en la rana dendrobátida $C$. machalilla y en la rana marsupial G. riobambae $(7,14)$.

En $X$. laevis, las células que involucionan hacia el interior de la gástrula se alejan de los labios del blastoporo por migración activa del mesodermo de la cabeza y por el proceso de CED del mesodermo del tronco (15). En C. machalilla se ha demostrado que el mesodermo de la cabeza se aleja de los labios del blastoporo durante la gastrulación. En tanto el mesodermo del tronco permanece en los labios del blastoporo hasta completar la gastrulación, y forman un collar circumblastoporal prominente. Solamente entonces se inicia la CED que al alargar el notocordio promueve el movimiento de las células que estaban concentradas en el gran collar circumblastoporal (15).

En $X$. laevis, la CED se inicia en la gástrula media solapando el proceso de alargamiento del cuerpo con el proceso de gastrulación. Una situación similar se presenta en la rana túngara $E$. randi (15). Ambas especies gozan de desarrollo rápido y el notocordio se alarga debido a los movimientos de CED durante la gastrulación. En contraste, en las ranas de desarrollo más lento, C. machalilla y G. riobambae, la CED está disociada de la gastrulación y el alargamiento del cuerpo se inicia una vez que concluye la misma. De modo similar, en E. anthonyi se ha reportado que el alargamiento del notocordio ocurre una vez que concluye la gastrulación (14). La comparación señala que la gástrula de $E$. anthonyi comparte las características de la gástrula de C. machalilla. Ambas especies tienen desarrollo lento en comparación con $X$. laevis.

Conclusiones y perspectivas.- El estudio de la gastrulación en E. anthon$y i$ brinda información adicional sobre la modularidad de la gastrulación en ranas 


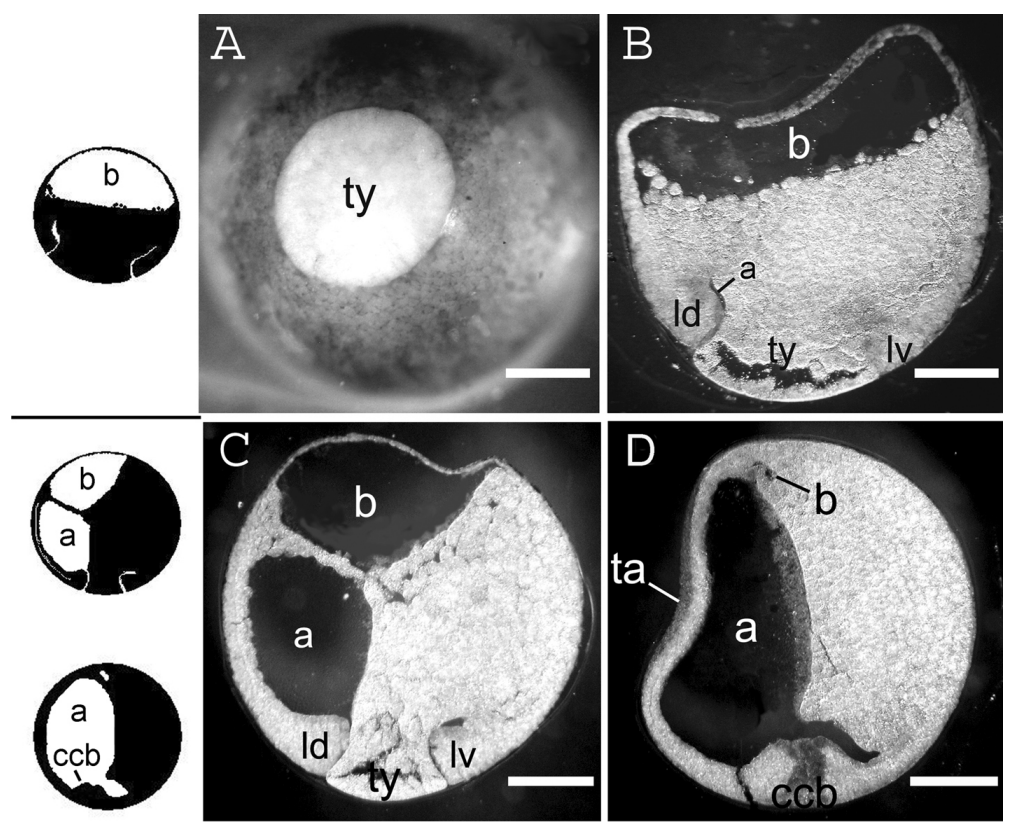

Figura 1. Morfología de la gástrula de E. antonyi. (A) Vista externa de una gástrula media. (B) Sección sagital de una gástrula media. Hay un artefacto de procesamiento en el tapón de yema (C) Sección sagital de una gástrula avanzada. (D) Sección sagital de una gástrula muy avanzada. Los dibujos a la izquierda muestran el avance en el tamaño del arquenterón durante la gastrulación. El arquenterón permanece pequeño hasta la gástrula avanzada, cuando se inicia su expansión. Abreviaturas: a, arquenterón; b, blastocele; ccb, collar circumblastoporal; ld, labio dorsal del blastoporo; lv, labio ventral del blastoporo; ta, techo del arquenterón; ty, tapón de yema. Las barras corresponden a $500 \mu \mathrm{m}$ en A, C y D y $400 \mu \mathrm{m}$ en B.

$(7,8,14)$ y da un nuevo ejemplo de la separación del proceso de gastrulación de la CED. Dicha separación va asociada con retardo en el alargamiento del arquenterón, concentración de células en los labios del blastoporo y la formación de un collar circumblastoporal prominente. El alargamiento del notocordio se ha retardado hasta después de culminar la gastrulación (14), lo que señala que el alargamiento del cuerpo está retardado en esta especie, al igual que en C. machalilla y $G$. riobambae, ranas con reproducción terrestre en nidos terrestres $\mathrm{y}$ en la bolsa materna, respectivamente $(7,14)$. Celeridad en el alargamiento del cuerpo, en cambio se presenta en aque- llas ranas cuyos huevos están expuestos al ambiente acuático como $X$. laevis o en el nido de espuma que flota en el agua de $E$. randi. Un rápido alargamiento del cuerpo se requeriría para la rápida formación del renacuajo, el mismo que al tener movimiento puede protegerse de depredadores. Tal presión ambiental por alargar el tronco está ausente en los huevos de ranas con modos terrestres de reproducción como $C$. machalilla y $E$. anthonyi y en la rana marsupial $G$. riobambae (8). La gran diversidad de modos reproductivos de las ranas ecuatorianas estudiadas está asociada con cambios en la gastrulación. Dichas modificaciones representan experimentos 
naturales, cuyo estudio ayuda a comprender mejor el desarrollo embrionario. Al mismo tiempo tales análisis nos ayudan documentar mejor la biología de especies de ranas ecuatorianas.

\section{AGRADECIMIENTOS}

Se expresa agradecimiento O. D. Pérez, I. M. Moya, M. Venegas-Ferrín, N. Sáenz-Ponce y demás colaboradores del Laboratorio de Biología del Desarrollo de la Pontificia Universidad Católica del Ecuador (PUCE) por su ayuda durante la realización de este estudio. Se agradece a la Mtr. M. Rivera y a los miembros del Laboratorio de Herpetología de la PUCE y su programa "Balsa de los sapos" por la donación de posturas de E. anthonyi. Este trabajo recibió el apoyo de becas de investigación de la PUCE.

\section{REFERENCIAS}

\section{BIBLIOGRÁFICAS}

1. GRANT, T.; FROST D. R.; CALDWELL. J. P.; GAGLIARDO, R.; HADDAD, C. F. B.; KOK, P. J. R.; MEANS, D. B.; NOONAN, B. P.; SCHARGEL, W. E. \& WHEELER, W. C. 2006. Phylogenetic systematics of dart-poison frogs and their relatives (Amphibia: Athesphatanura: Dendrobatidae). Bulletin American Museum Natural History, 299: 1-262.

2. QCAZ, AmphibiaWebEcuador-Anura-Epipedobates anthonyi. Página de Internet : http://www.puce.edu.ec/ zoologia/vertebrados/amphibiawe- bec/especies/anura/dendrobatidae/ anthonyi/index.html Consultada 18-abril-2011.

3. ZIMMERMANN, H. \& ZIMMERMANN, E. (1987). Mindestanforderungen für eine artgerechte Haltung einiger tropischer Anurenarten. Zeitschrift des Kölner Zoo, 30: 6171.

4. DALY, J. W.; GARRAFFO, H. M.; SPANDE, T. F.; DECKER, M. W.; SULLIVAN, J. P. \& WILLIAMS, M. 2000. Alkaloids from frog skin: the discovery of epibatidine and the potential for developing novel nonopioid analgesics. Natural Product Reports, 17: 131-135.

5. ZIMMERMANN, H. 1983. Durch Nachzucht erhalten: Phyllobates tricolor. Aquarien-Magazin, 17: 17-22.

6. DEL PINO, E. M.; ÁVILA, M. E.; PÉREZ, O. D.; BENÍTEZ, M. S.; ALARCÓN, I.; NOBOA, V. \& MOYA, I. M. 2004. Development of the dendrobatid frog Colostethus machalilla. International Journal of Developmental Biology, 48: 663-670.

7. DEL PINO, E. M.; VENEGAS-FERRÍN, M.; ROMERO-CARVAJAL, A.; MONTENEGRO-LARREA, P.; SÁENZ-PONCE, N.; MOYA, I. M.; ALARCÓN, I.; SUDOU, N.; YAMAMOTO, S. \& TAIRA, M. 2007. A comparative analysis of frog early development. Proceedings of the National Academy of Sciences U. S. A., 104: 11882-11888. 
8. DEL PINO, E. M. 2010. La gastrulación en ranas con diversos modos de reproducción. Revista Ecuatoriana de Medicina y Ciencias Biológicas, Casa de la Cultura Ecuatoriana, Quito, 31: 94-105.

9. KELLER, R. \& SHOOK, D. 2004. Gastrulation in amphibians. En: STERN, C. ed. Gastrulation: From cells to embryo: 171-203. Cold Spring Harbor Laboratory Press. New York.

10. DEL PINO, E. M. \& ELINSON R. P. 1983. Gastrulation produces an embryonic disc, a novel developmental pattern for frogs. Nature, 306: 589-591.

11. BECKHAM, Y. M.; NATH, K. \& ELINSON, R. P. 2003. Localization of RNAs in oocytes of Eleutherodactylus coqui, a direct developing frog, differs from Xenopus laevis. Evolution and Development, 5: 562-571.

12. WOLPERT, L.; JESSELL, T.; LAWRENCE, P.; MEYEROWITZ, E.; ROBERSON, E. \& SMITH, J. 2007. Principles of development. Tercera Edición. Oxford University Press, Oxford, United Kingdom.

13. DEL PINO, E. M. 1996. The expression of Brachyury (T) during gastrulation in the marsupial frog Gastrotheca riobambae. Developmental Biology, 177: 64-72.

14. MOYA, I. M.; ALARCÓN, I. \& DEL PINO, E. M. 2007. Gastrulation of Gastrotheca riobambae in compari- son with other frogs. Developmental Biology, 304: 467-478.

15. VENEGAS-FERRÍN, M.; SUDOU, N.; TAIRA, M. \& DEL PINO, E. M. 2010. Comparison of Lim1 expression in embryos of frogs with different modes of reproduction. International Journal Developmental Biology, 54: 195-202.

16. DEL PINO, E. M, \& ESCOBAR B. 1981. Embryonic stages of Gastrotheca riobambae (Fowler) during maternal incubation and comparison of development with that of other egg-brooding hylid frogs. Journal of Morphology, 167: 277-295.

17. ELINSON, R. P. \& DEL PINO, E. M. (1985). Cleavage and gastrulation in the egg-brooding, marsupial frog, Gastrotheca riobambae. Journal of Embryology and Experimental Morphology, 90: 223-232.

18. ROMERO-CARVAJAL, A.; SÁENZPONCE, N.; VENEGAS-FERRÍN, M.; ALMEIDA-REINOSO, D.; LEE C.; BOND J.; RYAN M. J.; WALLINGFORD J. B. \& DEL PINO, E.M. 2009. Embryogenesis and Laboratory Maintenance of the Foam-Nesting Túngara Frogs, Genus Engystomops (= Physalaemus). Developmental Dynamics, 238: 1444-1454. 